\title{
THE COMMUTANT OF $L(H)$ IN ITS ULTRAPOWER MAY OR MAY NOT BE TRIVIAL
}

\author{
ILIJAS FARAH, N. CHRISTOPHER PHILLIPS, AND JURIS STEPRĀNS
}

\begin{abstract}
Kirchberg asked in 2004 whether the commutant of $L(H)$ in its (norm) ultrapower is trivial. Assuming the Continuum Hypothesis, we prove that the answer depends on the choice of the ultrafilter.
\end{abstract}

Let $H$ be a separable infinite dimensional complex Hilbert space, fixed throughout. The purpose of this paper is to prove that, assuming the Continuum Hypothesis, the commutant of $L(H)$ (the algebra of bounded linear operators on $H$ ) in its ultrapower depends on the choice of the ultrafilter. This provides a somewhat surprising - and somewhat incomplete - answer to Question 2.22 of [15].

We follow the convention that $0 \in \mathbb{N}$. Let $A$ be a $\mathrm{C}^{*}$-algebra, and let $\mathcal{V}$ be a nonprincipal ultrafilter on $\mathbb{N}$ (equivalently, a point in $\beta \mathbb{N} \backslash \mathbb{N}$ ). (In the operator algebra literature, nonprincipal ultrafilters are usually denoted $\omega$; since in the set theory literature $\omega$ is reserved for the least infinite ordinal we suppress using this symbol altogether.) We denote by $\ell^{\infty}(A)$ the $\mathrm{C}^{*}$-algebra of all bounded functions from $\mathbb{N}$ to $A$, and we denote by $A^{\mathcal{V}}$ the ultrapower of $A$ associated with $\mathcal{V}([11],[2])$. More explicitly, $A^{\mathcal{V}}$ is the quotient of $\ell^{\infty}(A)$ by the (two-sided, norm-closed, selfadjoint) ideal

$$
c_{\mathcal{V}}(A)=\left\{\left(a_{n}\right) \in \ell^{\infty}(A): \lim _{n \rightarrow \mathcal{V}}\left\|a_{n}\right\|=0\right\} .
$$

(This algebra is denoted $A_{\mathcal{V}}$ in [15] and [16].) We identify $A$ with the subalgebra of $A^{\mathcal{V}}$ consisting of all

$$
(a, a, a, \ldots)+c_{\mathcal{V}}(A)
$$

for $a \in A$, that is, the image in $A^{\mathcal{V}}$ of all constant sequences in $\ell^{\infty}(A)$. If $A \subseteq B$, we denote by $A^{\prime} \cap B$ the relative commutant of $A$ in $B$, that is

$$
A^{\prime} \cap B=\{b \in B: a b=b a \text { for all } a \in A\} .
$$

(By convention, $A^{\prime}$ is used only when $B$ is the algebra of bounded operators on a Hilbert space.) Following Kirchberg [15], we define the invariant $F_{\mathcal{V}}(A)$

Date: November 6, 2018.

1991 Mathematics Subject Classification. 46L05, 03E50.

Ilijas Farah and Juris Steprāns were partially supported by NSERC. N. Christopher Phillips was partially supported by NSF grant DMS-0701076, by the Fields Institute for Research in Mathematical Sciences, Toronto, Canada, and by an Elliott Distinguished Visitorship at the Fields Institute. 
of $A$ to be the quotient of $A^{\prime} \cap A^{\mathcal{V}}$ by the annihilator

$$
\operatorname{Ann}\left(A, A^{\mathcal{V}}\right)=\left\{b \in A^{\mathcal{V}}: b a=0 \text { for all } a \in A\right\} .
$$

If $A$ is unital then the annihilator is trivial and

$$
F_{\mathcal{V}}(A)=A^{\prime} \cap A^{\mathcal{V}}
$$

The algebra $F_{\mathcal{V}}(A)$ for unital $A$, often denoted $A_{\mathcal{V}}$ in the literature (see [12]), has played an important role in the study of $\mathrm{C}^{*}$-algebras, particularly in the classification of separable nuclear unital purely infinite simple $\mathrm{C}^{*}$-algebras and group actions on them. (See [14], [16], [22], and [12].) The most important result, proved for example in [16], is that if $A$ is a separable nuclear unital purely infinite simple $\mathrm{C}^{*}$-algebra, then $F_{\mathcal{V}}(A)$ is again a purely infinite simple $C^{*}$-algebra. See [11] for further discussion of the uses of algebras of this type, and [15] for more recent applications of $F_{\mathcal{V}}(A)$.

The use of $F_{\mathcal{V}}(A)$ for purely infinite simple $\mathrm{C}^{*}$-algebras parallels an older use of its tracial analog for factors of type $\mathrm{II}_{1}$. We briefly describe this use to give context, but it is not needed in the sequel. In place of operator norm convergence, one uses convergence in the $L^{2}$ norm derived from the trace in the definition of $c_{\mathcal{V}}(A)$. For example, if $M$ is the hyperfinite factor of type $\mathrm{II}_{1}$, then the tracial analog of $F_{\mathcal{V}}(M)$ is again a factor of type $\mathrm{II}_{1}$. (See Lemma XIV.4.5 and Theorems XIV.4.6 and XIV.4.18 of [26]. This is also in [3], and in [8] it was shown that this is a consequence of the Fundamental Theorem for Ultraproducts from logic.) As one example, this fact is used to prove that outer actions of suitable groups on the hyperfinite factor of type $\mathrm{II}_{1}$ have the Rokhlin property, in turn a key step in the classification of such actions. See [13] for the case of finite groups, and [21] for the case of countable amenable groups.

Assuming the Continuum Hypothesis, Ge and Hadwin proved (11, Corollary 3.4]) that if $A$ is separable, then the isomorphism class of $F \mathcal{V}(A)$ is independent of the choice of the nonprincipal ultrafilter $\mathcal{V}$. But $L(H)$ is not, of course, separable in the norm topology. (The easiest way to see this is to note that projections onto the closed subspaces spanned by all subsets of a fixed orthonormal basis form an uncountable discrete set.)

Let $K(H)$ denote the ideal of compact operators in $L(H)$. For the Calkin algebra $C(H)=L(H) / K(H)$, Kirchberg proved ([15, Corollary 2.21]) that $F_{\mathcal{V}}(C(H))=\mathbb{C}$. This also implies $F_{\mathcal{V}}(L(H)) \subseteq \mathbb{C}+K(H)^{\mathcal{V}}$. In 15, Question 2.22], Kirchberg asked whether $F_{\mathcal{V}}(L(H))=\mathbb{C}$. The answer to this question is somewhat surprising.

Theorem 1. There is a nonprincipal ultrafilter $\mathcal{V}$ such that $F_{\mathcal{V}}(L(H)) \neq \mathbb{C}$.

Proof. This follows from Theorem 3.3 and Theorem 4.1

Theorem 2. Assume the Continuum Hypothesis. Then there is a nonprincipal ultrafilter $\mathcal{V}$ such that $F_{\mathcal{V}}(L(H))=\mathbb{C}$. 
Proof. This follows from Corollary 2.4, where we prove $F_{\mathcal{V}}(L(H))=\mathbb{C}$ for a selective ultrafilter $\mathcal{V}$, and from the fact that the Continuum Hypothesis implies selective ultrafilters exist (Proposition 1.4).

We record a curious consequence of Theorem 1 and Theorem 2 .

Corollary 3. Assume the Continuum Hypothesis. Then the isomorphism type of $F_{\mathcal{V}}(L(H))$ depends on the choice of the ultrafilter $\mathcal{V}$.

It should be noted that in the absence of the Continuum Hypothesis, $F_{\mathcal{V}}(A)$ depends on the choice of $\mathcal{V}$ for every infinite-dimensional separable $\mathrm{C}^{*}$-algebra $A$ ([7] for real rank zero and $[8$ in the general case). We don't know whether some axiom beyond ZFC is needed for the conclusion of Corollary 3 but it should be noted that an assumption much weaker than Continuum Hypothesis suffices. As noted above, the Continuum Hypothesis implies that the isomorphism type of $F_{\mathcal{V}}(A)$ does not depend on $A$ for a separable $A$. The reason is that, under the Continuum Hypothesis, for any two nonprincipal ultrafilters $\mathcal{V}$ and $\mathcal{W}$ on $\mathbb{N}$ there is an isomorphism between $A^{\mathcal{V}}$ and $A^{\mathcal{W}}$ that sends $A$ to itself ([11, Theorem 3.1]). The latter fact is an immediate consequence of the fact, provable in Zermelo-Fraenkel set theory with the Axiom of Choice (ZFC), that the unit ball of $A^{\mathcal{V}}$ is countably saturated in the logic of metric structures. (See [9, §4.4].) Since the Continuum Hypothesis implies that the ultrapowers are of size $\aleph_{1}$, a back-and-forth construction easily gives an isomorphism between $A^{\mathcal{V}}$ and $A^{\mathcal{W}}$ that sends the copy of $A$ in one ultrapower to the copy of $A$ in the other ultrapower. (See [9] for definitions.)

One curiosity deserves a mention here. By the countable saturatedness of ultrapowers, the Continuum Hypothesis implies that $L(H)^{\mathcal{U}}$ and $L(H)^{\mathcal{V}}$ are isomorphic for any two nonprincipal ultrafilters $\mathcal{U}$ and $\mathcal{V}$ on $\mathbb{N}$. However, if $\mathcal{U}$ is selective and $\mathcal{V}$ is flat, then by Corollary 3 we have $F_{\mathcal{V}}(L(H)) \neq F_{\mathcal{U}}(L(H))$ and therefore no isomorphism between $L(H)^{\mathcal{U}}$ and $L(H)^{\mathcal{V}}$ sends $L(H)$ to $L(H)$.

Acknowledgments. We would like to thank Eberhard Kirchberg for useful conversations. The results of this paper were obtained during the Fall 2007 Fields Institute semester on operator algebras and the May 2008 Canadian Operator Symposium in Toronto.

\section{Selective ultrafilters}

Definition 1.1. Let $M$ be a $C^{*}$-algebra or a von Neumann algebra. We let $[a, b]$ denote the additive commutator, $a b-b a$. Following [15] we say that a norm-bounded sequence $\left(a_{n}\right)_{n \in \mathbb{N}}$ in $M$ is central if for every $b \in M$ we have $\lim _{n \rightarrow \infty}\left\|\left[a_{n}, b\right]\right\|=0$. A sequence $\left(a_{n}\right)_{n \in \mathbb{N}}$ is trivial if

$$
\lim _{n \rightarrow \infty} \inf _{\lambda \in \mathbb{C}}\left\|a_{n}-\lambda\right\|=0 .
$$

(Note that such sequences are always central.) If $\mathcal{V}$ is an ultrafilter on $\mathbb{N}$ then a norm-bounded sequence $\left(a_{n}\right)_{n \in \mathbb{N}}$ is a $\mathcal{V}$-central sequence if for every 
$b \in M$ we have $\lim _{n \rightarrow \mathcal{V}}\left\|\left[a_{n}, b\right]\right\|=0$. A $\mathcal{V}$-central sequence $\left(a_{n}\right)_{n \in \mathbb{N}}$ is trivial if $\lim _{n \rightarrow \mathcal{V}} \inf _{\lambda \in \mathbb{C}}\left\|a_{n}-\lambda\right\|=0$.

Note that we have defined norm central sequences and norm trivial central sequences, even in the case of a von Neumann algebra. These are not the same as the central sequences usually considered for a type $\mathrm{II}_{1}$ factor.

Notation 1.2. If $X$ is a set then $[X]^{2}$ denotes the set of all two-element subsets of $X$ and $[X]^{\infty}$ denotes the set of all infinite subsets of $X$. We consider the space $[\mathbb{N}]^{\infty}$ with the Polish (that is, separable and completely metrizable) topology inherited from the Cantor set (identified with the power set of $\mathbb{N}$ ).

Recall that a subset of a Polish space is analytic if it is a continuous image of a Borel subset of a Polish space. The following result is well-known, but we sketch a proof of the easy implications for convenience of the reader.

Theorem 1.3 (A. R. D. Mathias). The following are equivalent for an ultrafilter $\mathcal{V}$ on $\mathbb{N}$.

(1) If $X_{0} \supseteq X_{1} \supseteq X_{2} \supseteq \ldots$ are in $\mathcal{V}$ then there exists $X \in \mathcal{V}$ such $X \backslash\{0,1, \ldots, n\} \subseteq X_{n}$ for every $n \in X$.

(2) For every $g: \mathbb{N} \rightarrow \mathbb{N}$ there is $A \in \mathcal{V}$ such that $g\lceil A$ is either constant or injective.

(3) For every $E \subseteq[\mathbb{N}]^{2}$ there is $A \in \mathcal{V}$ such that either $[A]^{2} \subseteq E$ or $[A]^{2} \cap E=\varnothing$.

(4) For every analytic $\mathbb{E} \subseteq[\mathbb{N}]^{\infty}$ there is $A \in \mathcal{V}$ such that either $[A]^{\infty} \subseteq$ $\mathbb{E}$ or $[A]^{\infty} \cap \mathbb{E}=\varnothing$.

Proof. The fact that (1) implies (4) is Mathias's theorem [19, Theorem 0.13]. We include proofs of the easy implications for the convenience of the reader.

We consider (4) implies (3). Let $E \subseteq[\mathbb{N}]^{2}$. Let $\mathbb{E} \subseteq[\mathbb{N}]^{\infty}$ be the set of all $X \in[\mathbb{N}]^{\infty}$ such that the pair consisting of the least two elements of $X$ is in $E$. Then $\mathbb{E}$ is analytic (and even open).

For (3) implies (2), given $g$ let $\{m, n\} \in E$ if and only if $g(m) \neq g(n)$.

For (2) implies (1), we may assume $X_{0}=\mathbb{N}$ and $\bigcap_{n=0}^{\infty} X_{n}=\varnothing$. Define $g(k)=n$ if $k \in X_{n} \backslash X_{n+1}$. Since $\mathcal{V}$ contains no set on which $g$ is constant, (2) implies that there is $Y \in \mathcal{V}$ such that $g$ is injective on $Y$.

Recursively find $0=m_{0}<m_{1}<\cdots$ so that for all $k$ and all $j \geq m_{k+1}$ in $Y$, we have $g(j) \geq m_{k}$. Let $g_{1}: \mathbb{N} \rightarrow \mathbb{N}$ be defined by $g_{1}(j)=k$ if $m_{k} \leq j<m_{k+1}$. Let $Z \subseteq Y$ be a set in $\mathcal{V}$ on which $g_{1}$ is injective.

Assume for the moment that $X=Z \cap \bigcup_{k=0}^{\infty}\left[m_{2 k}, m_{2 k+1}\right)$ is in $\mathcal{V}$. For $n \in X$ let $k$ be the unique integer such that $m_{2 k} \leq n<m_{2 k+1}$. Then $X \backslash\{0,1, \ldots, n\}$ is disjoint from $\left\{0,1, \ldots, m_{2 k+2}-1\right\}$. Therefore for $m \in$ $X \backslash\{0,1, \ldots, n\}$ we have $g(m) \geq m_{2 k+1}>n$. Hence $m \in X_{n}$ as required.

If $Z \cap \bigcup_{k=0}^{\infty}\left[m_{2 k}, m_{2 k+1}\right) \notin \mathcal{V}$, we instead set $X=Z \cap \bigcup_{k=0}^{\infty}\left[m_{2 k+1}, m_{2 k+2}\right)$. This set is in $\mathcal{V}$ and an argument analogous to the above shows that $X \backslash$ $\{0,1, \ldots, n\} \subseteq X_{n}$ for all $n \in X$. 
An ultrafilter satisfying the conditions of Theorem 1.3 is said to be selective (or Ramsey, or even happy). More information on these remarkable objects can be found in [19] or [6]. (For example, see Section 5 and Theorem 4.9 of [6].)

It is well-known that the existence of selective ultrafilters can be deduced from the Continuum Hypothesis ([19, Proposition 0.11]). We sketch a proof of this fact for the convenience of the reader.

Proposition 1.4. Assume the Continuum Hypothesis. Then there exists a nonprincipal selective ultrafilter.

Proof. Let $\omega_{1}$ be the first uncountable ordinal. Use the Continuum Hypothesis to enumerate all functions $g: \mathbb{N} \rightarrow \mathbb{N}$ as $g_{\gamma}$, for $\gamma<\omega_{1}$. We claim that there are infinite sets $A_{\gamma}$ for $\gamma<\omega_{1}$ such that the following holds for $\gamma<\omega_{1}$ :

(1) $g_{\gamma}$ is either constant or injective on $A_{\gamma}$.

(2) $A_{\gamma} \backslash A_{\delta}$ is finite if $\delta<\gamma$.

We prove the claim by transfinite induction. If $g_{0}$ is constant on an infinite subset of $\mathbb{N}$, let $A_{0}$ be this set. Otherwise, the image of $\mathbb{N}$ under $g_{0}$ is infinite and we can find an infinite subset $A_{0} \subseteq \mathbb{N}$ on which $g_{0}$ is injective.

Assume $A_{\gamma}$ has been chosen. Use the argument above, with $A_{\gamma}$ in place of $\mathbb{N}$ and $g_{\gamma+1}$ in place of $g_{0}$, to choose an infinite subset $A_{\gamma+1} \subseteq A_{\gamma}$ on which $g_{\gamma+1}$ is either constant or injective.

Now assume $\gamma$ is a limit ordinal and the sets $A_{\delta}$ have been chosen for $\delta<\gamma$. Re-enumerate the sets $A_{\delta}$ for $\delta<\gamma$ as $A_{j}^{\prime}$ for $j \in \mathbb{N}$. For $j \in \mathbb{N}$ let

$$
\eta=\max \left(\left\{\delta<\gamma: A_{\delta}=A_{k}^{\prime} \text { for some } k<j\right\}\right) .
$$

Then the set $A_{\eta} \backslash \bigcap_{k<j} A_{k}^{\prime}$ is finite, and in particular $\bigcap_{k<j} A_{k}^{\prime}$ is infinite for every $j$. We can therefore choose a sequence $n_{0}<n_{1}<n_{2}<\cdots$ so that $n_{j} \in \bigcap_{k<j} A_{k}^{\prime}$ for all $j \in \mathbb{N}$. Set $B=\left\{n_{j}: j \in \mathbb{N}\right\}$. Then $B$ is infinite and $B \backslash A_{\delta}$ is finite for all $\delta<\gamma$. Now $A_{\gamma} \subseteq B$ is chosen as above.

The family $\left\{A_{\gamma}\right\}_{\gamma \in \omega_{1}}$ has the finite intersection property - indeed, for any finite set $F \subseteq \omega_{1}$, if $\gamma=\max (F)$ then there is a finite set $a \subseteq A_{\gamma}$ such that $A_{\gamma} \backslash a \subseteq \bigcap_{\delta \in F} A_{\delta}$. Hence this family can be extended to an ultrafilter, and this ultrafilter is selective by construction.

For $\mathbf{a} \in A^{\mathcal{V}}$ a sequence $\left(a_{n}\right)_{n \in \mathbb{N}}$ in $\ell^{\infty}(A)$ such that $\mathbf{a}=\left(a_{n}\right)_{n \in \mathbb{N}}+c_{\mathcal{V}}(A)$ is called a representing sequence. A von Neumann algebra $M$ is separably acting if it is isomorphic to a weak operator closed selfadjoint subalgebra of the bounded operators on a separable Hilbert space. Thus $L(H)$, for our fixed infinite dimensional separable Hilbert space $H$, is separably acting.

Theorem 1.5. Assume $M$ is a separably acting von Neumann algebra and $\mathcal{V}$ is a selective ultrafilter. Then for $\mathbf{a} \in M^{\mathcal{V}}$ the following are equivalent.

(1) $\mathbf{a} \in M^{\prime} \cap M^{\mathcal{V}}$.

(2) a has a representing sequence that is a central sequence. 
Proof. We need only prove that (11) implies (2). Fix a $\in M^{\mathcal{V}}$ and a representing sequence $\left(a_{n}\right)_{n \in \mathbb{N}}$.

The closed unit ball $\mathbf{B}$ of $M$ with the weak operator topology is compact metric, and is therefore a Polish space. See page 35 of [4]. For the convenience of the reader, we sketch the proof here.

We may assume $M \subseteq L(H)$ for our fixed infinite dimensional separable Hilbert space $H$. Let $D \subseteq H$ be a countable norm dense subset, and let $C \subseteq$ $\mathbb{C}$ be a countable dense subset. Observe that the weak operator topology on $\mathbf{B}$ has a base consisting of sets of the form

$$
\bigcap_{j=1}^{n}\left\{a \in \mathbf{B}:\left|\left\langle a \xi_{j}, \eta_{j}\right\rangle-\lambda_{j}\right|<1 / k_{j}\right\}
$$

with $n \in \mathbb{N}$ and

$\xi_{1}, \xi_{2}, \ldots, \xi_{n}, \eta_{1}, \eta_{2}, \ldots, \eta_{n} \in D, \lambda_{1}, \lambda_{2}, \ldots, \lambda_{n} \in C$, and $k_{1}, k_{2}, \ldots, k_{n} \in \mathbb{N}$.

So $\mathbf{B}$ with the weak operator topology is second countable. To see that it is a Polish space use the Banach-Alaoglu theorem to conclude that it is, in fact, compact.

The power set $\mathcal{P}(\mathbb{N})$ with the Cantor set topology is compact and metrizable and, hence, also a Polish space.

We will prove that the set

$$
\mathbb{E}=\left\{X \in[\mathbb{N}]^{\infty}:(\exists j \in \mathbb{N})(\exists b \in \mathbf{B})(\forall n \in X)\left\|\left[a_{n}, b\right]\right\|>1 / j\right\}
$$

is analytic.

For each $\varepsilon>0$ define $\Phi_{\varepsilon}: \mathbf{B} \times \mathcal{P}(\mathbb{N}) \rightarrow \mathcal{P}(\mathbb{N})$ by

$$
\Phi_{\varepsilon}(b, X)=\left\{n \in X:\left\|\left[a_{n}, b\right]\right\|>\varepsilon\right\}
$$

for $b \in \mathbf{B}$ and $X \subseteq \mathbb{N}$. We claim that $\Phi_{\varepsilon}$ is Borel measurable. First, since multiplication is separately weak operator continuous and norm closed balls in $M$ are also weak operator closed, for each fixed $n$ the set

$$
J_{n}=\left\{b \in \mathbf{B} \times \mathcal{P}(\mathbb{N}):\left\|\left[a_{n}, b\right]\right\|>\varepsilon\right\}
$$

is open in B. Now let $U \subseteq \mathcal{P}(\mathbb{N})$ be a basic open set, that is, there are $N \in \mathbb{N}$ and a subset $S \subseteq\{0,1, \ldots, N\}$ such that $U$ consists of all $X \subseteq \mathbb{N}$ with $X \cap\{0,1, \ldots, N\}=S$. Then

$$
\begin{aligned}
\Phi_{\varepsilon}^{-1}(U)=\{(b, X) \in \mathbf{B} \times \mathcal{P}(\mathbb{N}): & S=X \cap\{0,1, \ldots, N\}\} \\
& \cap\left(\bigcap_{n \in S} J_{n}\right) \cap\left(\bigcap_{n \in\{0,1, \ldots, N\} \backslash S} \mathbf{B} \backslash J_{n}\right),
\end{aligned}
$$

establishing that $\Phi_{\varepsilon}^{-1}(U)$ is Borel. This proves that $\Phi_{\varepsilon}$ is Borel measurable.

Define $Z=\coprod_{j=1}^{\infty}[\mathbf{B} \times \mathcal{P}(\mathbb{N})]$, and define $\Phi: Z \rightarrow \mathcal{P}(\mathbb{N})$ by letting $\Phi=\Phi_{1 / j}$ on the $j$ component of the disjoint union. Since $M$ acts on a separable Hilbert space, $Z$ is a Polish space. Clearly $\Phi$ is Borel. The subset $[\mathbb{N}]^{\infty} \subseteq$ 
$\mathcal{P}(\mathbb{N})$ is Borel, since its complement is countable. Therefore $\Phi^{-1}\left([\mathbb{N}]^{\infty}\right)$ is Borel and the set

$$
\mathbb{E}=\Phi\left(\Phi^{-1}\left([\mathbb{N}]^{\infty}\right)\right)=[\mathbb{N}]^{\infty} \cap \bigcup_{j=1}^{\infty} \Phi_{1 / j}[\mathbf{B} \times \mathcal{P}(\mathbb{N})]
$$

is analytic. Since $\mathcal{V}$ is selective, there is $X \in \mathcal{V}$ such that $[X]^{\infty} \subseteq \mathbb{E}$ or $[X]^{\infty} \cap \mathbb{E}=\varnothing$.

Let us first assume the second possibility applies. Then for each $b \in \mathbf{B}$ and $j \in \mathbb{N}$ with $j>0$, the set $\left\{n \in X:\left\|\left[a_{n}, b\right]\right\|>1 / j\right\}$ is not in $\mathbb{E}$, and therefore must be finite. Let $a_{n}^{\prime}=a_{n}$ if $n \in X$ and $a_{n}^{\prime}=1$ if $n \notin X$. Then $\left\{n \in \mathbb{N}:\left\|\left[a_{n}^{\prime}, b\right]\right\|>\varepsilon\right\}$ is finite for all $b \in \mathbf{B}$ and $\varepsilon>0$, so $\left(a_{n}^{\prime}\right)_{n \in \mathbb{N}}$ is a central sequence. It represents a since $X \in \mathcal{V}$.

Now assume there is $X \in \mathcal{V}$ such that $[X]^{\infty} \subseteq \mathbb{E}$. In particular, there are $b \in \mathbf{B}$ and $j \in \mathbb{N}$ such that $\left\|\left[a_{n}, b\right]\right\| \geq 1 / j$ for all $n \in X$; therefore $\mathbf{a} \notin M^{\prime} \cap M^{\mathcal{V}}$.

Corollary 1.6. Assume $M$ is a separably acting von Neumann algebra. If $\mathcal{V}$ is selective then the following are equivalent.

(1) There exists a nontrivial central sequence in $M$.

(2) There exists a nontrivial $\mathcal{V}$-central sequence in $M$.

Proof. Assume $\left(a_{n}\right)_{n \in \mathbb{N}}$ is a nontrivial central sequence. By passing to a subsequence, we may assume there is $\varepsilon>0$ such that $\inf _{\lambda \in \mathbb{C}}\left\|a_{n}-\lambda\right\| \geq$ $\varepsilon$ for all $n$. This sequence is clearly a nontrivial $\mathcal{V}$-central sequence for any nonprincipal ultrafilter $\mathcal{V}$. The converse implication is an immediate consequence of Theorem 1.5 .

Let $M$ be a type $\mathrm{II}_{1}$ factor with (unique) tracial state $\tau$. Let $\|\cdot\|_{2}$ be the standard $L^{2}$-norm on $M$, defined by $\|a\|_{2}=\sqrt{\tau\left(a^{*} a\right)}$. A bounded sequence $\left(a_{n}\right)$ in $M$ is tracially central if $\lim _{n}\left\|\left[b, a_{n}\right]\right\|_{2}=0$ for every $b \in M$. (This, rather than that of Definition 1.1, is the usual definition of a central sequence in this context.) The tracial ultrapower of a $\mathrm{II}_{1}$ factor is the ultrapower of the metric structure $\left(M,\|\cdot\|_{2}\right)$ in the sense of [2]. Analogues of Theorem 1.5 and Corollary 1.6 for tracial ultrapowers of $\mathrm{II}_{1}$ factors could be proved by mimicking the above proofs. However, in this context the assumption that $\mathcal{V}$ is a selective ultrafilter can be dropped. In fact, for every nonprincipal ultrafilter $\mathcal{V}$ on $\mathbb{N}$, the commutant of $M$ in $M^{\mathcal{V}}$ is trivial if and only if $M$ has no nontrivial central sequences. By an observation of McDuff ([20, remark after Lemma 5]) this follows by a diagonalization argument from the fact that the metric $\|\cdot\|_{2}$ on a $\mathrm{II}_{1}$ factor is separable. Similarly, the analogues of Theorem 1.5 and Corollary 1.6 hold when $M$ is a separable $\mathrm{C}^{*}$-algebra and $\mathcal{V}$ is any nonprincipal ultrafilter on $\mathbb{N}$.

On the other hand, Theorem 1 and Theorem 2.1 below imply that some assumption on $\mathcal{V}$ in Theorem 1.5 and Corollary 1.6 is necessary in the case when $M=L(H)$. 


\section{No NONTRIVIAL CENTRAL SEQUENCES}

Let $M$ be a von Neumann algebra with center $\mathcal{Z}(M)$. In 25, Proposition 3.1], David Sherman proved that a sequence $\left(a_{n}\right)_{n \in \mathbb{N}}$ in $M$ is norm central if and only if $\lim _{n \rightarrow \infty} \inf _{z \in \mathcal{Z}(M)}\left\|a_{n}-z\right\|=0$. In particular, if $M$ is a factor then it has no nontrivial central sequences. Note that we are referring to norm central sequences, even if $M$ is a $\mathrm{II}_{1}$-factor. For the reader's convenience we provide a self-contained proof of the special case of Sherman's result needed in the proof of Theorem 2 ,

Theorem 2.1. There are no nontrivial central sequences in $L(H)$.

The proof of Theorem 2.1 will be given after two lemmas.

Lemma 2.2. Let $c \in L(H)$ be a selfadjoint operator that is not a scalar. Set $\delta=\inf _{\lambda \in \mathbb{C}}\|c-\lambda\|$. Then $\delta>0$, and for every $\varepsilon>0$ there is a rank one partial isometry $s \in L(H)$ such that $\|[s, c]\|>2 \delta-\varepsilon$.

Proof. We denote the spectrum of $c$ by $\operatorname{sp}(c)$. Set $\lambda_{1}=\inf (\operatorname{sp}(c))$ and $\lambda_{2}=\sup (\operatorname{sp}(c))$. By considering the isomorphism between $C(\operatorname{sp}(c))$ and the $\mathrm{C}^{*}$-subalgebra of $L(H)$ generated by $c$ and 1 , we see that $\delta=\frac{1}{2}\left(\lambda_{2}-\lambda_{1}\right)>0$. Set

$$
\varepsilon_{0}=\frac{\varepsilon}{4\|c\|+2}
$$

Choose $\varepsilon_{1}>0$ so small that if $\alpha \in \mathbb{R}$ satisfies $|\alpha-1|<\varepsilon_{1} \delta^{-1}$, then $\left|\alpha^{-1}-1\right|<$ $\varepsilon_{0}$. We also require $\varepsilon_{1} \leq \min \left(\varepsilon_{0}, \delta \varepsilon_{0}\right)$.

Choose $\xi_{1}, \eta \in H$ with

$$
\left\|\xi_{1}\right\|=\|\eta\|=1, \quad\left\|c \xi_{1}-\lambda_{1} \xi_{1}\right\|<\varepsilon_{1}, \quad \text { and } \quad\left\|c \eta-\lambda_{2} \eta\right\|<\varepsilon_{1} .
$$

Set

$$
\mu=\eta-\left\langle\eta, \xi_{1}\right\rangle \xi_{1} \quad \text { and } \quad \xi_{2}=\|\mu\|^{-1} \mu
$$

Then $\left\|\xi_{2}\right\|=1$ and $\left\langle\xi_{1}, \xi_{2}\right\rangle=0$.

We need to estimate $\left\|c \xi_{2}-\lambda_{2} \xi_{2}\right\|$. We begin as follows, using $\left\langle\xi_{1}, c \eta\right\rangle=$ $\left\langle c \xi_{1}, \eta\right\rangle$ at the second step:

$$
\begin{aligned}
\left|\left(\lambda_{2}-\lambda_{1}\right)\left\langle\xi_{1}, \eta\right\rangle\right| & =\left|\left\langle\xi_{1}, \lambda_{2} \eta\right\rangle-\left\langle\lambda_{1} \xi_{1}, \eta\right\rangle\right| \\
& =\left|\left\langle\xi_{1}, \lambda_{2} \eta-c \eta\right\rangle+\left\langle c \xi_{1}-\lambda_{1} \xi_{1}, \eta\right\rangle\right| \\
& \leq\left\|\xi_{1}\right\| \cdot\left\|\lambda_{2} \eta-c \eta\right\|+\left\|\lambda_{1} \xi_{1}-c \xi_{1}\right\| \cdot\|\eta\|<2 \varepsilon_{1} .
\end{aligned}
$$

It follows that

$$
\begin{gathered}
\|\mu-\eta\|=\left|\left\langle\xi_{1}, \eta\right\rangle\right|<\frac{2 \varepsilon_{1}}{\lambda_{2}-\lambda_{1}}=\varepsilon_{1} \delta^{-1}, \\
\text { so }|1-\|\mu\||<\varepsilon_{1} \delta^{-1} \text {, and } \\
\left\|\xi_{2}-\eta\right\| \leq\|\| \mu\left\|^{-1} \mu-\mu\right\|+\|\mu-\eta\|<\varepsilon_{0}+\varepsilon_{1} \delta^{-1} \leq 2 \varepsilon_{0}
\end{gathered}
$$

Therefore

$$
\left\|c \xi_{2}-\lambda_{2} \xi_{2}\right\|<2 \varepsilon_{0}\|c\|+\left\|c \eta-\lambda_{2} \eta\right\|+2 \varepsilon_{0}\left|\lambda_{2}\right| \leq(4\|c\|+1) \varepsilon_{0} .
$$


Now let $s \in L(H)$ be the partial isometry such that $s \xi_{1}=\xi_{2}$ and $s \xi=0$ whenever $\left\langle\xi, \xi_{1}\right\rangle=0$. Then

$$
\left\|s c \xi_{1}-\lambda_{1} \xi_{2}\right\| \leq\|s\| \cdot\left\|c \xi_{1}-\lambda_{1} \xi_{1}\right\|<\varepsilon_{1} \leq \varepsilon_{0} .
$$

So

$$
\begin{aligned}
\|[s, c]\| & \geq\left\|s c \xi_{1}-c s \xi_{1}\right\|=\left\|s c \xi_{1}-c \xi_{2}\right\| \\
& \geq\left(\lambda_{2}-\lambda_{1}\right)-\left\|\lambda_{1} \xi_{2}-s c \xi_{1}\right\|-\left\|\lambda_{2} \xi_{2}-c \xi_{2}\right\| \\
& >2 \delta-\varepsilon_{0}-(4\|c\|+1) \varepsilon_{0} \geq 2 \delta-\varepsilon .
\end{aligned}
$$

This completes the proof.

Lemma 2.3. Let $A$ be a unital $C^{*}$-algebra and let $a \in A$. Then

$$
\inf _{\lambda \in \mathbb{C}}\|a-\lambda\| \geq \frac{1}{2}(\|a\|-\| \text { pap } \|)
$$

for every nonzero projection $p$ in $A$.

Proof. Let $\lambda \in \mathbb{C}$. We have

$$
\|a-\lambda\| \geq\|a\|-|\lambda| .
$$

Also, using $p \neq 0$ at the third step,

$$
\|a-\lambda\| \geq\|p(a-\lambda) p\|=\|p a p-\lambda p\| \geq|\lambda|-\| \text { pap } \| .
$$

Therefore

$$
\|a-\lambda\| \geq \frac{1}{2}(\|a\|-|\lambda|)+\frac{1}{2}(|\lambda|-\|p a p\|)=\frac{1}{2}(\|a\|-\|p a p\|),
$$

as desired.

Proof of Theorem 2.1. Using the decomposition of an operator $a$ as $a=$ $\frac{1}{2}\left(a+a^{*}\right)+\frac{1}{2}\left(a-a^{*}\right)$, with $\frac{1}{2}\left(a+a^{*}\right)$ and $-\frac{i}{2}\left(a-a^{*}\right)$ selfadjoint, we need only prove that there are no nontrivial selfadjoint central sequences. Let $\left(a_{j}\right)_{j \in \mathbb{N}}$ be a norm bounded sequence of selfadjoint elements of $L(H)$ (not necessarily central) which is not trivial in the sense of Definition 1.1. We prove that $\left(a_{j}\right)_{j \in \mathbb{N}}$ is not central. It suffices to find a subsequence which is not central. By passing to a subsequence, we may assume

$$
\inf _{j \in \mathbb{N}} \inf _{\lambda \in \mathbb{C}}\left\|a_{j}-\lambda\right\|>0 .
$$

In particular, our sequence $\left(a_{j}\right)_{j \in \mathbb{N}}$ has no subsequence which converges in norm to any element of $\mathbb{C} \cdot 1$.

We will find $\varepsilon>0$, an element $b \in L(H)$, and a subsequence $\left(a_{m(j)}\right)_{j \in \mathbb{N}}$ of $\left(a_{j}\right)_{j \in \mathbb{N}}$, such that $\left\|\left[a_{m(j)}, b\right]\right\|>\varepsilon$ for all $j \in \mathbb{N}$. This will show that $\left(a_{j}\right)_{j \in \mathbb{N}}$ is not central, and prove the theorem.

Let $\left(r_{n}\right)_{n \in \mathbb{N}}$ be a strictly increasing sequence of finite rank projections such that $\lim _{n \rightarrow \infty} r_{n}=1$ in the strong operator topology, and with $r_{0}=0$.

First suppose that there are $n \in \mathbb{N}$, a number $\delta>0$, and a subsequence $\left(a_{l(j)}\right)_{j \in \mathbb{N}}$ of $\left(a_{j}\right)_{j \in \mathbb{N}}$, such that

$$
\inf _{j \in \mathbb{N}} \inf _{\lambda \in \mathbb{C}}\left\|r_{n} a_{l(j)} r_{n}-\lambda r_{n}\right\| \geq \delta .
$$


Since $r_{n}$ has finite rank, there is a further subsequence $\left(a_{m(j)}\right)_{j \in \mathbb{N}}$ of $\left(a_{l(j)}\right)_{j \in \mathbb{N}}$ such that $c=\lim _{j \rightarrow \infty} r_{n} a_{m(j)} r_{n}$ exists. Then also $\inf _{\lambda \in \mathbb{C}}\left\|c-\lambda r_{n}\right\| \geq \delta$. Lemma 2.2 provides $s \in r_{n} L(H) r_{n}$ such that $\|[s, c]\|>\frac{3}{2} \delta$. Then

$$
\left\|\left[s, a_{m(j)}\right]\right\|=\left\|\left[s, r_{n} a_{m(j)} r_{n}\right]\right\|>\delta
$$

for all sufficiently large $j$. Dropping initial terms of the subsequence $\left(a_{m(j)}\right)_{j \in \mathbb{N}}$, we obtain the required subsequence with $b=s$ and $\varepsilon=\delta$.

Accordingly, we may now assume that

$$
\lim _{j \rightarrow \infty} \inf _{\lambda \in \mathbb{C}}\left\|r_{n} a_{j} r_{n}-\lambda r_{n}\right\|=0
$$

for all $n \in \mathbb{N}$.

Set $M=\sup _{j \in \mathbb{N}}\left\|a_{j}\right\|$. Then

$$
\lim _{j \rightarrow \infty} \inf _{\lambda \in[-M, M]}\left\|r_{n} a_{j} r_{n}-\lambda r_{n}\right\|=0
$$

for all $n \in \mathbb{N}$. In particular, there are numbers $\lambda_{0, j} \in[-M, M]$ such that

$$
\lim _{j \rightarrow \infty}\left\|r_{0} a_{j} r_{0}-\lambda_{0, j} r_{0}\right\|=0 .
$$

By compactness, there are $\lambda_{0} \in[-M, M]$ and a strictly increasing sequence $\left(l_{0}(j)\right)_{j \in \mathbb{N}}$ such that $\lim _{j \rightarrow \infty} \lambda_{0, l_{0}(j)}=\lambda_{0}$. Then

$$
\lim _{j \rightarrow \infty}\left\|r_{0} a_{j} r_{0}-\lambda_{0} r_{0}\right\|=0 .
$$

Similarly, there are $\lambda_{1, j} \in[-M, M]$ such that

$$
\lim _{j \rightarrow \infty}\left\|r_{1} a_{l_{0}(j)} r_{1}-\lambda_{1, j} r_{1}\right\|=0,
$$

and then there are $\lambda_{1} \in[-M, M]$ and a subsequence $\left(l_{1}(j)\right)_{j \in \mathbb{N}}$ of $\left(l_{0}(j)\right)_{j \in \mathbb{N}}$ such that

$$
\lim _{j \rightarrow \infty}\left\|r_{1} a_{l_{1}(j)} r_{1}-\lambda_{1} r_{1}\right\|=0
$$

We proceed inductively, obtaining numbers $\lambda_{n} \in[-M, M]$ and subsequences $\left(l_{n}(j)\right)_{j \in \mathbb{N}}$ of $\left(l_{n-1}(j)\right)_{j \in \mathbb{N}}$ such that

$$
\lim _{j \rightarrow \infty}\left\|r_{n} a_{l_{n}(j)} r_{n}-\lambda_{n} r_{n}\right\|=0 .
$$

Setting $l(j)=l_{j}(j)$, we then get

$$
\lim _{j \rightarrow \infty}\left\|r_{n} a_{l(j)} r_{n}-\lambda_{n} r_{n}\right\|=0
$$

for all $n \in \mathbb{N}$.

Clearly $\lambda_{0}=\lambda_{1}=\cdots$. Subtracting this common value from each $a_{j}$ does not change the conditions on $\left(a_{j}\right)_{j \in \mathbb{N}}$ or the existence of the required subsequence, so without loss of generality $\lim _{j \rightarrow \infty}\left\|r_{n} a_{l(j)} r_{n}\right\|=0$ for all $n$.

Suppose now that there is $n \in \mathbb{N}$ such that $\left\|r_{n} a_{l(j)}\left(1-r_{n}\right)\right\|$ does not converge to 0 as $j \rightarrow \infty$. Then there are $\rho>0$ and a subsequence $\left(a_{m(j)}\right)_{j \in \mathbb{N}}$ 
of $\left(a_{l(j)}\right)_{j \in \mathbb{N}}$ such that $\left\|r_{n} a_{m(j)}\left(1-r_{n}\right)\right\|>\rho$ for all $j \in \mathbb{N}$. With $b=r_{n}$, and using $r_{n}\left(1-r_{n}\right)=0$ at the second step, we have

$$
\left\|\left[b, a_{m(j)}\right]\right\| \geq\left\|r_{n}\left[b, a_{m(j)}\right]\left(1-r_{n}\right)\right\|=\left\|r_{n} a_{m(j)}\left(1-r_{n}\right)\right\|>\rho
$$

for all $j \in \mathbb{N}$. Thus, we have a subsequence $\left(a_{m(j)}\right)_{j \in \mathbb{N}}$ of the required type with $b=r_{n}$ and $\varepsilon=\rho$.

Since $\left\|r_{n} a_{j}\left(1-r_{n}\right)\right\|=\left\|\left(1-r_{n}\right) a_{j} r_{n}\right\|$, we may assume without loss of generality that

$$
\lim _{j \rightarrow \infty}\left\|r_{n} a_{j}\left(1-r_{n}\right)\right\|=\lim _{j \rightarrow \infty}\left\|\left(1-r_{n}\right) a_{j} r_{n}\right\|=0
$$

for all $n \in \mathbb{N}$. Combining these with $\lim _{j \rightarrow \infty}\left\|r_{n} a_{j} r_{n}\right\|=0$ for all $n$, we have reduced to consideration of the case

$$
\lim _{j \rightarrow \infty}\left\|a_{j}-\left(1-r_{n}\right) a_{j}\left(1-r_{n}\right)\right\|=0
$$

for all $n \in \mathbb{N}$.

Since by assumption $\left(a_{j}\right)_{j \in \mathbb{N}}$ does not converge to 0 in norm, there are $\delta>0$ and a subsequence $\left(a_{l(j)}\right)_{j \in \mathbb{N}}$ of $\left(a_{j}\right)_{j \in \mathbb{N}}$ such that $\inf _{j \in \mathbb{N}}\left\|a_{l(j)}\right\| \geq$ $\delta$. Passing to this subsequence, without loss of generality $\inf _{j \in \mathbb{N}}\left\|a_{j}\right\| \geq \delta$. We now construct recursively a subsequence $\left(a_{m(j)}\right)_{j \in \mathbb{N}}$ of $\left(a_{j}\right)_{j \in \mathbb{N}}$, and an increasing sequence $(n(j))_{j \in \mathbb{N}}$ with $n(0)=0$, such that the elements

$$
y_{j}=\left(r_{n(j+1)}-r_{n(j)}\right) a_{m(j)}\left(r_{n(j+1)}-r_{n(j)}\right)
$$

satisfy

$$
\inf _{\lambda \in \mathbb{C}}\left\|y_{j}-\lambda\left(r_{n(j+1)}-r_{n(j)}\right)\right\|>\frac{1}{4} \delta
$$

for all $j \in \mathbb{N}$. We repeatedly use the observation that $\lim _{n \rightarrow \infty}\left\|r_{n} x r_{n}\right\|=\|x\|$ for all $x \in L(H)$.

Begin by choosing $n(0)=0$, so that $r_{n(0)}=0$. Choose $m(0) \in \mathbb{N}$ such that $\left\|r_{1} a_{m(0)} r_{1}\right\|<\frac{1}{4} \delta$. Now use $\left\|a_{m(0)}\right\| \geq \delta$ to choose $n(1)$ so large that the element $y_{0}=r_{n(1)} a_{m(0)} r_{n(1)}$ satisfies $\left\|y_{0}\right\|>\frac{3}{4} \delta$. Apply Lemma 2.3 with $A=r_{n(1)} L(H) r_{n(1)}$, with $a=y_{0}$, and with $p=r_{1}$, to get

$$
\inf _{\lambda \in \mathbb{C}}\left\|y_{0}-\lambda r_{n(1)}\right\|>\frac{1}{2}\left(\frac{3}{4} \delta-\frac{1}{4} \delta\right)=\frac{1}{4} \delta .
$$

Given $m(j)$ and $n(j+1)$, choose $m(j+1)>m(j)$ so large that, with

$$
x=\left(1-r_{n(j+1)+1}\right) a_{m(j+1)}\left(1-r_{n(j+1)+1}\right),
$$

we have $\left\|a_{m(j+1)}-x\right\|<\frac{1}{6} \delta$. Then choose $n(j+2)$ so large that

$$
\left\|r_{n(j+2)} x r_{n(j+2)}\right\|>\|x\|-\frac{1}{6} \delta \text {. }
$$

Set

Then

$$
y_{j+1}=\left(r_{n(j+2)}-r_{n(j+1)}\right) a_{m(j+1)}\left(r_{n(j+2)}-r_{n(j+1)}\right) \text {. }
$$

$$
\begin{aligned}
\left\|y_{j+1}\right\| & \geq\left\|\left(r_{n(j+2)}-r_{n(j+1)+1}\right) a_{m(j+1)}\left(r_{n(j+2)}-r_{n(j+1)+1}\right)\right\| \\
& =\left\|r_{n(j+2)} x r_{n(j+2)}\right\|>\|x\|-\frac{1}{6} \delta>\left\|a_{m(j+1)}\right\|-\frac{1}{6} \delta-\frac{1}{6} \delta \geq \frac{2}{3} \delta .
\end{aligned}
$$


Also,

$$
\left\|r_{n(j+1)+1} y_{j+1} r_{n(j+1)+1}\right\| \leq\left\|r_{n(j+1)+1}\left(a_{m(j+1)}-x\right) r_{n(j+1)+1}\right\|<\frac{1}{6} \delta .
$$

Apply Lemma 2.3 with

$$
A=\left(r_{n(j+2)}-r_{n(j+1)}\right) L(H)\left(r_{n(j+2)}-r_{n(j+1)}\right),
$$

with $a=y_{j+1}$, and with $p=r_{n(j+1)+1}-r_{n(j+1)}$, to get

$$
\inf _{\lambda \in \mathbb{C}}\left\|y_{j+1}-\lambda\left(r_{n(j+2)}-r_{n(j+1)}\right)\right\|>\frac{1}{2}\left(\frac{2}{3} \delta-\frac{1}{6} \delta\right)=\frac{1}{4} \delta
$$

as desired. This completes the construction of $\left(a_{m(j)}\right)_{j \in \mathbb{N}}$ and $(n(j))_{j \in \mathbb{N}}$.

Now let $y_{j}$ be as in (11) for $j \in \mathbb{N}$. Lemma 2.2 provides

$$
s_{j} \in\left(r_{n(j+1)}-r_{n(j)}\right) L(H)\left(r_{n(j+1)}-r_{n(j)}\right)
$$

such that $\left\|\left[s_{j}, y_{j}\right]\right\|>\frac{1}{4} \delta$ and $\left\|s_{j}\right\|=1$. The series $s=\sum_{j=0}^{\infty} s_{j}$ converges in the strong operator topology, and for $j \in \mathbb{N}$ we have

$$
\left\|\left[s, a_{m(j)}\right]\right\| \geq\left\|\left(r_{n(j+1)}-r_{n(j)}\right)\left[s, a_{m(j)}\right]\left(r_{n(j+1)}-r_{n(j)}\right)\right\|=\left\|\left[s_{j}, y_{j}\right]\right\|>\frac{1}{4} \delta .
$$

Thus, the subsequence $\left(a_{m(j)}\right)_{j \in \mathbb{N}}$ satisfies the required condition with $b=s$ and $\varepsilon=\frac{1}{4} \delta$.

The following is an immediate consequence of Corollary [1.6 and Theorem 2.1.

Corollary 2.4. If $\mathcal{V}$ is a selective ultrafilter then $F \mathcal{V}(L(H))=\mathbb{C}$.

\section{Flat ultrafilters}

Notation 3.1. By $f: \mathbb{N} \nearrow \mathbb{N}$ we mean that $f$ is a strictly increasing function from $\mathbb{N}$ to $\mathbb{N}$ such that $f(0)>0$.

For such $f$ and nonincreasing $h: \mathbb{N} \rightarrow[0,1]$ the assertion $\|h-h \circ f\|_{\infty} \leq \varepsilon$ is equivalent to stating that the variation of $h$ on any interval of the form $\mathbb{N} \cap[j, f(j)]$ is at most $\varepsilon$.

Definition 3.2. An ultrafilter $\mathcal{V}$ on $\mathbb{N}$ is flat if there are nonincreasing functions $h_{n}: \mathbb{N} \rightarrow[0,1]$, for $n \in \mathbb{N}$, such that:

(1) $h_{n}(0)=1$ for all $n \in \mathbb{N}$.

(2) $\lim _{j \rightarrow \infty} h_{n}(j)=0$ for all $n \in \mathbb{N}$.

(3) For every $f: \mathbb{N} \nearrow \mathbb{N}$, we have $\lim _{n \rightarrow \mathcal{V}}\left\|h_{n}-h_{n} \circ f\right\|_{\infty}=0$.

Theorem 3.3. Flat ultrafilters exist.

We need a lemma.

Lemma 3.4. Let $f: \mathbb{N} \nearrow \mathbb{N}$. Let $n \in \mathbb{N}$ with $n>0$, let $m_{0}=0$, and suppose $m_{l+1} \geq f\left(m_{l}\right)$ for $0<l \leq n$. Set

$$
h=\sum_{l=0}^{n} \frac{n-l}{n} \chi_{\mathbb{N} \cap\left[m_{l}, m_{l+1}\right)} .
$$

Then $\|h-h \circ f\|_{\infty} \leq 1 / n$. 
Proof. Fix $j \in \mathbb{N}$. If $j \geq m_{n+1}$ then $h(j)=0=h \circ f(j)$. Otherwise there is $l$ such that $m_{l} \leq j<m_{l+1}$. Then $f(j)<f\left(m_{l+1}\right) \leq m_{l+2}$ (writing $\left.m_{n+2}=\infty\right)$. Since $h$ is nonincreasing,

$$
\frac{n-l}{n}=h(j) \geq h \circ f(j) \geq \frac{n-l-1}{n} .
$$

The required estimate is now clear.

Proof of Theorem 3.3. Let $\mathbb{F}$ be the countable set of all nonincreasing functions $h: \mathbb{N} \rightarrow \mathbb{Q} \cap[0,1]$ that are eventually zero and such that $h(0)=1$. We start by constructing an ultrafilter $\mathcal{V}$ on $\mathbb{F}$. For $f: \mathbb{N} \nearrow \mathbb{N}$ and $\varepsilon>0$ let

$$
X_{f, \varepsilon}=\left\{h \in \mathbb{F}:\|h-h \circ f\|_{\infty} \leq \varepsilon\right\} .
$$

By Lemma 3.4 this set is infinite. On the other hand,

$$
X_{f, \varepsilon} \cap X_{g, \delta} \supseteq X_{\max (f, g), \min (\varepsilon, \delta)} .
$$

Therefore the collection of all $X_{f, \varepsilon}$, for $f: \mathbb{N} \nearrow \mathbb{N}$ and $\varepsilon>0$, has the finite intersection property. Let $\mathcal{W}$ be any ultrafilter which extends this collection.

Let $k: \mathbb{N} \rightarrow \mathbb{F}$ be a bijection, and set $\mathcal{V}=\{A \subseteq \mathbb{N}: k(A) \in \mathcal{W}\}$, which is an ultrafilter on $\mathbb{N}$. We claim that $\mathcal{V}$ is flat. The functions $h_{n}$ required in the definition are given by $h_{n}=k(n)$ for $n \in \mathbb{N}$. Conditions (11) and (2) in Definition 3.2 are immediate. For Condition (3), let $f: \mathbb{N} \nearrow \mathbb{N}$ and let $\varepsilon>0$. Then $Y=k^{-1}\left(X_{f, \varepsilon}\right) \in \mathcal{V}$, and for $n \in Y$ we have $h_{n} \in X_{f, \varepsilon}$ by construction, so that $\left\|h_{n}-h_{n} \circ f\right\|_{\infty} \leq \varepsilon$. This proves (3) in Definition 3.2.

\section{Nontrivial RELATive COMmUtants}

The present section is devoted to the proof of the following result.

Theorem 4.1. If $\mathcal{V}$ is a flat ultrafilter then $F_{\mathcal{V}}(L(H)) \neq \mathbb{C}$.

Notation 4.2. Fix an orthonormal basis $\left(\xi_{n}\right)_{n \in \mathbb{N}}$ for our separable infinitedimensional complex Hilbert space $H$, and let $e_{n}$ be the orthogonal projection onto $\mathbb{C} \xi_{n}$. Let $\mathbb{D}$ be the set of all nonincreasing functions $h: \mathbb{N} \rightarrow[0,1]$ such that $h(0)=1$ and $\lim _{n \rightarrow \infty} h(n)=0$. For $h \in \mathbb{D}$ define a compact operator $a_{h}$ (with $\left\|a_{h}\right\|=1$ since $h(0)=1$ ) by

$$
a_{h}=\sum_{j=0}^{\infty} h(j) e_{j} .
$$

Notation 4.3. Let $\vec{E}=\left(E_{n}\right)_{n \in \mathbb{N}}$ be a family of closed orthogonal subspaces of $H$ such that $H=\bigoplus_{n=0}^{\infty} E_{n}$. Let $\mathcal{D}(\vec{E})$ be the von Neumann algebra

$$
\left\{a \in L(H): a E_{n} \subseteq E_{n} \text { for all } n \in \mathbb{N}\right\} .
$$

For $f: \mathbb{N} \nearrow \mathbb{N}$ (as in Notation [3.1), and with $\left(\xi_{n}\right)_{n \in \mathbb{N}}$ as in Notation 4.2, let $f^{n}$ be the composite $f \circ f \circ \cdots \circ f$ (with $n$ terms), and take $f^{0}$ to be the constant function with value 0 . Define $\vec{E}^{f}$ by

$$
\vec{E}_{n}^{f}=\operatorname{span}\left\{\xi_{j}: f^{n}(0) \leq j<f^{n+1}(0)\right\},
$$


and set $\mathcal{D}(f)=\mathcal{D}\left(\vec{E}^{f}\right)$.

Lemma 4.4. Adopt Notation 4.2 and Notation 4.3. If $h \in \mathbb{D}, f: \mathbb{N} \nearrow \mathbb{N}$, and $\|h-h \circ f\|_{\infty} \leq \varepsilon$, then for every $b \in \mathcal{D}(f)$ we have $\left\|\left[a_{h}, b\right]\right\| \leq 2 \varepsilon\|b\|$.

Proof. Let $q_{n}$ be the orthogonal projection onto $E_{n}^{f}$. We can write

$$
a_{h}=\sum_{n=0}^{\infty} q_{n} a_{h} q_{n} .
$$

Define

$$
y=\sum_{n=0}^{\infty} h\left(f^{n}(0)\right) q_{n} .
$$

(Both series converge in norm because $\lim _{n \rightarrow \infty} h(n)=0$.) For any $n \in \mathbb{N}$ and for $f^{n}(0) \leq k<f^{n+1}(0)$, we have

$$
h\left(f^{n}(0)\right) \geq h(k) \geq h\left(f^{n+1}(0)\right) \geq h\left(f^{n}(0)\right)-\varepsilon,
$$

so $\left\|q_{n} a_{h} q_{n}-h\left(f^{n}(0)\right) q_{n}\right\| \leq \varepsilon$. Therefore $\left\|a_{h}-y\right\| \leq \varepsilon$. Since $y$ is a central element of $\mathcal{D}(f)$, the conclusion follows.

Lemma 4.5. Let $A$ be a unital $C^{*}$-algebra, let $e, f \in A$ be orthogonal projections, and let $a \in A$. Then

$$
\|e a e+e a f+f a e\| \leq 2\|a\| .
$$

Proof. We have

$$
e a e+e a f+f a e=(e+f) a(e+f)-f a f,
$$

and $\|(e+f) a(e+f)\|,\|f a f\| \leq\|a\|$.

Examples using $2 \times 2$ matrices show that it is not possible to replace the constant 2 in Lemma 4.5 by 1 , even if $a$ is selfadjoint.

The use of 'stratification' of $L(H)$ into von Neumann algebras $\mathcal{D}\left(g_{l}\right)$ as given in Lemma 4.7 below resembles the use in [5, Lemma 3.1], and the following lemma is a minor improvement to [5, Lemma 1.3].

Lemma 4.6. Let $F=\left\{a_{1}, a_{2}, \ldots, a_{k}\right\} \subset L(H)$ be finite, and let $\delta>0$. Then there exist $g_{0}, g_{1}: \mathbb{N} \nearrow \mathbb{N}$ and decompositions $a_{j}=a_{j}^{(0)}+a_{j}^{(1)}+c_{j}$ for $j=1,2, \ldots, k$, such that for $j=1,2, \ldots, k$ we have:

(1) $a_{j}^{(0)} \in \mathcal{D}\left(g_{0}\right)$.

(2) $a_{j}^{(1)} \in \mathcal{D}\left(g_{1}\right)$.

(3) $\left\|a_{j}^{(0)}\right\|,\left\|a_{j}^{(1)}\right\| \leq 2\left\|a_{j}\right\|$.

(4) $c_{j}$ is compact.

(5) $\left\|c_{j}\right\|<\delta$.

Proof. Let $p_{n}$ be the orthogonal projection onto $\operatorname{span}\left(\left\{\xi_{0}, \xi_{1}, \ldots, \xi_{n-1}\right\}\right)$. Thus $p_{0}=0$. Also choose $\rho_{0}, \rho_{1}, \ldots>0$ such that $2 \sum_{n=0}^{\infty} \rho_{n+1} \leq \delta$. 
We claim that there is a strictly increasing function $f: \mathbb{N} \rightarrow \mathbb{N}$ such that $f(0)=0$ and such that for every $n \in \mathbb{N}$ and every $a \in F$, we have

$$
\left\|\left(1-p_{f(n+1)}\right) a p_{f(n)}\right\|<\rho_{n} \text { and }\left\|p_{f(n)} a\left(1-p_{f(n+1)}\right)\right\|<\rho_{n} .
$$

(For $n=0$ the condition is vacuous because $p_{0}=0$.) We construct $f$ recursively. Start by taking $f(0)=0$. Given $f(n)$, use compactness of $p_{f(n)} a$ and $a p_{f(n)}$, finiteness of $F$, and the fact that $\left(p_{m}\right)_{m \in \mathbb{N}}$ is an approximate identity for $K(H)$, to choose $m>f(n)$ such that

$$
\left\|\left(1-p_{m}\right) a p_{f(n)}\right\|<\rho_{n} \text { and }\left\|p_{f(n)} a\left(1-p_{m}\right)\right\|<\rho_{n}
$$

for all $a \in F$. Then set $f(n+1)=m$. This proves the claim.

For $n \in \mathbb{N}$, we now set $q_{n}=p_{f(n+1)}-p_{f(n)}$. Since $p_{f(0)}=0$, the series $\sum_{n=0}^{\infty} q_{n}$ converges to 1 in the strong operator topology.

For $j=1,2, \ldots, k$, define, with convergence in the strong operator topology,

$$
a_{j}^{(0)}=\sum_{n=0}^{\infty}\left(q_{2 n} a_{j} q_{2 n}+q_{2 n} a_{j} q_{2 n+1}+q_{2 n+1} a_{j} q_{2 n}\right)
$$

and

$$
a_{j}^{(1)}=\sum_{n=0}^{\infty}\left(q_{2 n+1} a_{j} q_{2 n+1}+q_{2 n+1} a_{j} q_{2 n+2}+q_{2 n+2} a_{j} q_{2 n+1}\right) .
$$

The $n$th term in the series for $a_{j}^{(0)}$ is in $\left(q_{2 n}+q_{2 n+1}\right) L(H)\left(q_{2 n}+q_{2 n+1}\right)$, and the $n$th term in the series for $a_{j}^{(1)}$ is in $\left(q_{2 n+1}+q_{2 n+2}\right) L(H)\left(q_{2 n+1}+q_{2 n+2}\right)$. Accordingly, if for $j \in \mathbb{N}$ we set $g_{0}(j)=f(2 j+2)$ and $g_{1}(j)=f(2 j+1)$, then $g_{0}, g_{1}: \mathbb{N} \nearrow \mathbb{N}$ and parts (11) and (2) are satisfied. Part (3) follows from Lemma 4.5.

The estimates (2) give, for every $n \in \mathbb{N}$,

$$
\left\|q_{n} a_{j}\left(1-p_{f(n+2)}\right)\right\|=\left\|q_{n} p_{f(n+1)} a_{j}\left(1-p_{f(n+2)}\right)\right\|<\rho_{n+1},
$$

and similarly

Therefore the series

$$
\left\|\left(1-p_{f(n+2)}\right) a_{j} q_{n}\right\|<\rho_{n+1} .
$$

$$
\sum_{n=0}^{\infty}\left[q_{n} a_{j}\left(1-p_{f(n+2)}\right)+\left(1-p_{f(n+2)}\right) a_{j} q_{n}\right]
$$

converges in norm to a compact operator $c_{j}$ with

$$
\left\|c_{j}\right\|<2 \sum_{n=0}^{\infty} \rho_{n+1} \leq \delta
$$

This is parts (44) and (5). Also, $a_{j}^{(0)}+a_{j}^{(1)}+c_{j}=a_{j}$ is clear.

Lemma 4.7. Let $\mathcal{V}$ be an arbitrary ultrafilter on $\mathbb{N}$. For $\mathbf{a} \in L(H)^{\mathcal{V}}$ the following are equivalent:

(1) $\mathbf{a} \in L(H)^{\prime} \cap L(H)^{\mathcal{V}}$. 
(2) $\mathbf{a} \in \bigcap_{f: \mathbb{N} \nearrow \mathbb{N}}\left[\mathcal{D}(f)^{\prime} \cap L(H)^{\mathcal{V}}\right]$.

Proof. The implication from (11) to (2) is trivial.

Assume (2) and fix $\mathbf{b} \in L(H)$. Fix $\delta>0$. By Lemma 4.6 we can find $g_{0}, g_{1}: \mathbb{N} \nearrow \mathbb{N}$ and a decomposition $\mathbf{b}=\mathbf{b}_{0}+\mathbf{b}_{1}+\mathbf{c}$ such that $\mathbf{b}_{j} \in \mathcal{D}\left(g_{j}\right)$ for $j=0,1$ and $\|\mathbf{c}\| \leq \frac{1}{2} \delta$. Thus $[\mathbf{a}, \mathbf{b}]=\left[\mathbf{a}, \mathbf{b}_{0}+\mathbf{b}_{1}+\mathbf{c}\right]=[\mathbf{a}, \mathbf{c}]$ and therefore $\|[\mathbf{a}, \mathbf{b}]\| \leq \delta\|\mathbf{a}\|$. Since $\mathbf{b} \in L(H)$ and $\delta>0$ were arbitrary, $\mathbf{a} \in$ $L(H)^{\prime} \cap L(H)^{\mathcal{V}}$.

Proof of Theorem 4.1. Fix a sequence $\left(h_{n}\right)_{n \in \mathbb{N}}$ of functions witnessing the flatness of $\mathcal{V}$. Let $a_{n}=a_{h_{n}}=\sum_{j=0}^{\infty} h_{n}(j) e_{n}$, as in Notation 4.2. Fix $f: \mathbb{N} \nearrow \mathbb{N}$. Since $\lim _{n \rightarrow \mathcal{V}}\left\|h_{n}-h_{n} \circ f\right\|_{\infty}=0$, by Lemma 4.4 the sequence $\left(a_{n}\right)_{n \in \mathbb{N}}$ is a representing sequence of an element a of $\mathcal{D}(f)^{\prime} \cap L(H)^{\mathcal{V}}$. Since $f: \mathbb{N} \nearrow \mathbb{N}$ was arbitrary, by Lemma 4.7 we have $\mathbf{a} \in L(H)^{\prime} \cap L(H)^{\mathcal{V}}$.

Therefore $\left(a_{n}\right)_{n \in \mathbb{N}}$ is a $\mathcal{V}$-central sequence. Since each $a_{n}$ is compact and has norm one, this sequence is nontrivial.

\section{CONCluding Remarks}

The following is what remains of Kirchberg's question.

Question 5.1. Does there exist a nonprincipal ultrafilter $\mathcal{V}$ on $\mathbb{N}$ such that $F_{\mathcal{V}}(L(H))=\mathbb{C}$ ?

By our Theorem 2, the Continuum Hypothesis implies a positive answer, but the question is whether such an ultrafilter can be constructed in ZFC. A 'typical' statement independent from ZFC is decided by the Continuum Hypothesis or a strengthening such as Jensen's diamond principle in one way and by Martin's Axiom or a strengthening such as the Proper Forcing Axiom in another way. (See [18, Chapter II] for an introduction to Martin's Axiom.) An example in theory of operator algebras is the statement 'the Calkin algebra has an outer automorphism,' which follows from the Continuum Hypothesis ([23]) and is incompatible with a consequence of the Proper Forcing Axiom ([5]). This, however, is not the case with Question 5.1. It is well-known that (a rather weak form of) Martin's Axiom implies the existence of selective ultrafilters, and therefore the existence of $\mathcal{U}$ such that $F_{\mathcal{U}}(L(H))=\mathbb{C}$. A closer look at the proof of Proposition 1.4 reveals that it goes through when the Continuum Hypothesis is weakened to the assertion that for every family $\mathcal{F} \subseteq[\mathbb{N}]^{\infty}$ such that the intersection of any finitely many sets in $\mathcal{F}$ is infinite, and such that $|\mathcal{F}|<2^{\aleph_{0}}$, there is $B \in[\mathbb{N}]^{\infty}$ such that $B \backslash A$ is finite for all $A \in \mathcal{F}$. This assertion (known as $\mathfrak{p}=2^{\aleph_{0}}$ ) is an easy consequence of Martin's Axiom. (See [1, Section 7].)

By a result of Kunen ([17]), if ZFC is consistent then so is the theory 'ZFC + there are no selective ultrafilters'. However, in Kunen's model there exists an ultrafilter $\mathcal{V}$ such that $F_{\mathcal{V}}(L(H))=\mathbb{C}$. An ultrafilter $\mathcal{V}$ is a P-point if for every $g: \mathbb{N} \rightarrow \mathbb{N}$ there is $A \in \mathcal{V}$ such that $g$ is either constant or finite-to-one on $A$. In [10] it is proved that if $\mathcal{V}$ is a P-point then $F_{\mathcal{V}}(L(H))=\mathbb{C}$. While 
P-points exist in Kunen's model, Shelah has proved that if ZFC is consistent then so is ZFC + 'there are no P-points'. (See [24.)

We could not resolve the following question.

Question 5.2. If $\mathcal{V}$ is an ultrafilter such that $F \mathcal{V}(L(H)) \neq \mathbb{C}$, does it follow that $\mathcal{V}$ is flat?

As pointed out in the introduction, tools from the logic of metric structures $([2])$ are very relevant to the study of ultrapowers of $\mathrm{C}^{*}$-algebras. (See [8] for recent applications.) For example, it would be interesting to reformulate some of the results of [15] using the language of model theory. In particular, can the notion of $\sigma$-sub-Stonean ([15, Definition 1.4]) be replaced with the notion of $\aleph_{1}$-saturated ([2, Definition 7.5], the case when $\kappa=\aleph_{1}$, the least uncountable cardinal)?

\section{REFERENCES}

[1] A. Blass, Combinatorial cardinal characteristics of the continuum, to appear in Handbook of Set Theory, M. Foreman, M. Magidor, and A. Kanamori, editors. Available at http://www.math.lsa.umich.edu/ ablass/set.html

[2] I. Ben Yaacov, A. Berenstein, C.W. Henson, and A. Usvyatsov, Model theory for metric structures, in Model Theory with Applications to Algebra and Analysis, Vol. II (Z. Chatzidakis et al., eds.), Lecture Notes series of the London Math. Society, no. 350, Cambridge University Press, 2008, pp. 315-427.

[3] A. Connes, Outer conjugacy classes of automorphisms of factors, Ann. Sci. Éc. Norm. Sup. Sér. 4, 8 (1975), 383-420.

[4] J. Dixmier, Von Neumann Algebras, North-Holland, Amsterdam, New York, Oxford, 1981.

[5] I. Farah, All automorphisms of the Calkin algebra are inner, preprint (arXiv: 0705.3085v7 [math.OA]).

[6] I. Farah, Semiselective coideals, Mathematika 45 (1998), 79-103.

[7] I. Farah, The relative commutant of separable $C^{*}$-algebras of real rank zero, J. Funct. Anal. 256 (2009), 3841-3846.

[8] I. Farah, B. Hart, and D. Sherman, Model theory of operator algebras I: Stability, preprint (arXiv: 0908.2790v1 [math.OA]).

[9] I. Farah, B. Hart, and D. Sherman, Model theory of operator algebras II: Model theory, in preparation, 2009.

[10] I. Farah and J. Steprāns, Flat ultrafilters, in preparation, 2009.

[11] L. Ge and D. Hadwin, Ultraproducts of $C^{*}$-algebras, Recent advances in operator theory and related topics (Szeged, 1999), Oper. Theory Adv. Appl., vol. 127, Birkhäuser, Basel, 2001, pp. 305-326.

[12] M. Izumi, Finite group actions on $C^{*}$-algebras with the Rohlin property. I, Duke Math. J. 122 (2004), 233-280.

[13] V. F. R. Jones, Actions of finite groups on the hyperfinite type $I I_{1}$ factor, Mem. Amer. Math. Soc. 28 (1980), no. 237.

[14] E. Kirchberg, The classification of purely infinite $C^{*}$-algebras using Kasparov's theory, preliminary preprint (3rd draft).

[15] E. Kirchberg, Central sequences in $C^{*}$-algebras and strongly purely infinite algebras, Operator Algebras: The Abel Symposium 2004, Abel Symp., vol. 1, Springer, Berlin, 2006, pp. 175-231.

[16] E. Kirchberg and N. C. Phillips, Embedding of exact $C^{*}$-algebras in the Cuntz algebra $\mathcal{O}_{2}$, J. reine angew. Math. 525 (2000), 17-53. 
[17] K. Kunen, Some points in $\beta N$, Math. Proc. Cambridge Philos. Soc. 80 (1976), no. 3, $385-398$.

[18] K. Kunen, Set Theory: An Introduction to Independence Proofs, North-Holland, Amsterdam, 1980.

[19] A. R. D. Mathias, Happy families, Annals of Mathematical Logic 12 (1977), 59-111.

[20] D. McDuff, Central sequences and the hyperfinite factor, Proc. London Math. Soc. 21 (1970), 443-461.

[21] A. Ocneanu, Actions of Discrete Amenable Groups on von Neumann Algebras, Springer-Verlag Lecture Notes in Math. no. 1138, Springer-Verlag, Berlin, 1985.

[22] N. C. Phillips, A classification theorem for nuclear purely infinite simple $C^{*}$-algebras, Documenta Math. 5 (2000), 49-114 (electronic).

[23] N. C. Phillips and N. Weaver, The Calkin algebra has outer automorphisms, Duke Math. J. 139 (2007), 185-202.

[24] S. Shelah, Proper and Improper Forcing, 2nd. ed., Perspectives in Mathematical Logic, Springer-Verlag, Berlin, 1998.

[25] D. Sherman, Divisible operators in von Neumann algebras, Illinois J. Math., to appear.

[26] M. Takesaki, Theory of Operator Algebras III, Springer-Verlag, Berlin, etc., 2003.

Department of Mathematics and Statistics, York University, 4700 Keele Street, North York, Ontario, Canada, M3J 1P3, and Matematicki Institut, Kneza Mihaila 34, Belgrade, Serbia

URL: http://www.math.yorku.ca/ ifarah

E-mail address: ifarah@mathstat.yorku.ca

Department of Mathematics, University of Oregon, Eugene, Oregon 974031222, U.S.A.

$U R L$ : http: //www. uoregon. edu/ ncp/

E-mail address: ncp@uoregon.edu

Fields Institute, 222 College Street, Toronto, On, Canada

E-mail address: steprans@fields.utoronto.ca 